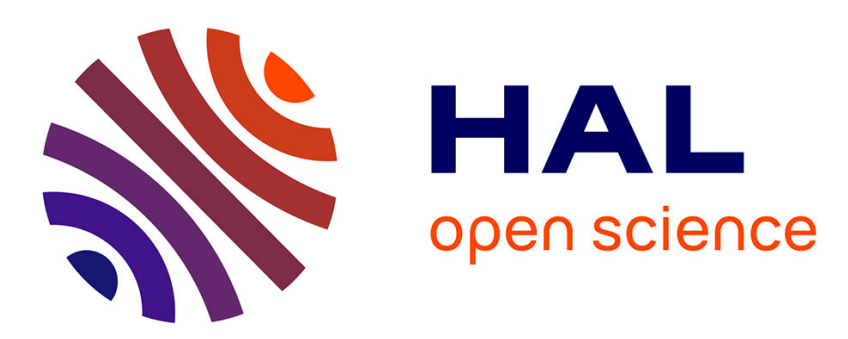

\title{
Sorting and Extraction of Self-Propelled Chiral Particles by Polarized Wall Currents
}

Thomas Barois, Jean-François Boudet, Juho S. Lintuvuori, Hamid Kellay

\section{To cite this version:}

Thomas Barois, Jean-François Boudet, Juho S. Lintuvuori, Hamid Kellay. Sorting and Extraction of Self-Propelled Chiral Particles by Polarized Wall Currents. Physical Review Letters, 2020, 125 (23), pp.238003. 10.1103/PhysRevLett.125.238003 . hal-03036916

\section{HAL Id: hal-03036916 https://hal.science/hal-03036916}

Submitted on 2 Dec 2020

HAL is a multi-disciplinary open access archive for the deposit and dissemination of scientific research documents, whether they are published or not. The documents may come from teaching and research institutions in France or abroad, or from public or private research centers.
L'archive ouverte pluridisciplinaire HAL, est destinée au dépôt et à la diffusion de documents scientifiques de niveau recherche, publiés ou non, émanant des établissements d'enseignement et de recherche français ou étrangers, des laboratoires publics ou privés. 


\title{
Sorting and Extraction of Self-Propelled Chiral Particles by Polarized Wall Currents
}

\author{
Thomas Barois@, Jean-François Boudet $\odot$, Juho S. Lintuvuori®, and Hamid Kellay $\bullet$ \\ Univ. Bordeaux, CNRS, LOMA, UMR 5798, F-33400 Talence, France
}

(Received 29 June 2020; revised 24 September 2020; accepted 23 October 2020; published 1 December 2020)

\begin{abstract}
The dynamics of self-propelled particles with curved trajectories is investigated. Two modes are observed, a bulk mode with a quasicircular motion and a surface mode with the particles following the walls. The surface mode is the only mode of ballistic transport and the particle current is polar and depends on the particles' chirality. We show that a robust sorting and extraction occurs when the particles explore a domain with two exit gates collecting selectively the particles circling left and right. With a counterslope, the extraction rate is found to increase while the sorting error is reduced.
\end{abstract}

DOI: 10.1103/PhysRevLett.125.238003

Collections of self-propelled particles [1-3] have attracted much attention as a minimal framework to mimic collective behavior observed in biological systems [4-6]. Such active assemblies are studied as a model situation for out-of-equilibrium physics in which the key ingredient is the directional motion of the particles. A recent development in active matter concerns the use of chiral particles. Two classes of chiral particles may be identified with, first, the spinners [7-14], meaning rotating elements without directional motion, and second, the circling particles [15-24] combining directional and rotational activity.

In this Letter, we take advantage of the polarized edge currents existing close to a confining surface for circling particles. We show that the wall current is robust in dilute regime, at much lower densities than what is observed with spinner assemblies. Our results demonstrate a process of polar separation within a binary mixture of particles circling either left or right. The sorted particles are not only phase separated [24-29] but also separated by types in two distinct compartments. This compartment sorting is spontaneously initiated by events of particle-particle collisions. It demonstrates that the source of orientational disorder from the collisions can induce a positional order with the polar separation in the target compartments. Finally, we demonstrate that the sorting can be tuned using an external field: by placing the two compartments at a higher altitude, the sorting rate and the accuracy of the sorting can be increased. As discussed below, this counterintuitive result can be understood by considering the role of the particle-particle collisions in the sorting process.

The interaction of an active particle with a wall is a key ingredient of this work. With nonchiral particles, the interaction with walls may induce particle sorting in various geometries such as funnels [30,31], wedges [32,33], or even flat walls [34]. The wall-confining surface interaction is also important in a biological context. For example, the interaction of a bacterium with walls may induce biased turns in branched channels [35], circular motion near walls
[36], or upstream swimming [37]. The interaction with boundaries plays also a central role in the migration of sperm cells [38] or the focusing of swimming algae by curvature gradients [39]. Concerning collective effects, the presence of walls induces quasi-2D clustering in the case of gliding bacteria [40]. The confinement of a bacterial suspension in a circular domain may also form large-scale circulations [41]. In model systems of active particles, the interaction with walls may be responsible for the guiding of artificial swimmers $[42,43]$ or the formation of surface clusters [44-46]. For chiral active particles, the interaction with walls may result in a biased wall flow that depends on the polarity of the particles. Polarized wall flows have been obtained in simulations of spinners in the form of monomers [9] and dimers [10]. Similar observations have also been reported for self-propelled particles with circular trajectories in experiments [13] and in simulations [15,17].

The chiral particles are electromechanical robots of two types, moving in curved trajectories turning either left or right. Figure 1(a) shows the trajectory of a right-turning particle in a bulk mode, far from any domain boundary. The radius of curvature of the particle's trajectory is of the order of the particle's length. The average period of the circular trajectory for a particle is $\langle T\rangle=1.33 \mathrm{~s}$ (see Supplemental Material, I [47]). This circular trajectory is similar to the trajectories obtained with asymmetric [48-50] or symmetric [51] particles and in simulations of selfpropelled particles with an added torque [16,19-21,23]. Figure 1(b) shows a second trajectory for the same righthanded particle guided by the wall in a counterclockwise surface mode.

During the trajectory recording of Fig. 1(c), a total of nine transitions from a bulk mode to a surface mode were identified. All the transitions occurred after a particleparticle collision: six transitions were subsequent to a collision involving two particles in bulk mode and the three other transitions were from a particle in bulk mode colliding with a particle in surface mode. A video (movie 
(a)

(c)

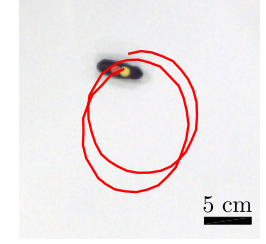

(b)

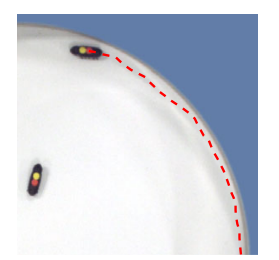

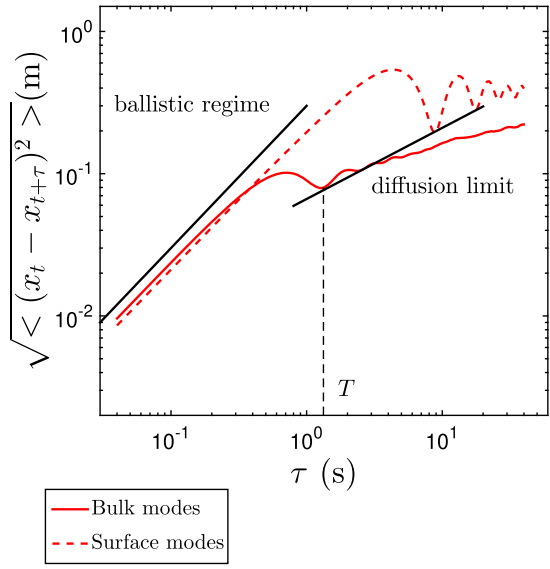

FIG. 1. (a) Trajectory of an isolated right-turning particle (2.6 s). (b) Trajectory of the same particle in surface mode. (c) Root mean square displacement (RMSD) for seven particles in a circular box as a function of the time increment. The solid line is the RMSD averaged over the particles that are not in contact with the walls. The dashed line is the RMSD for the particles close to the wall. A ballistic regime with a slope 1 and a diffusive regime with a slope $1 / 2$ are also represented (logarithmic axes).

v1) in the Supplemental Material [47] shows one of the transitions for a particle from bulk mode to surface mode after a particle-particle collision. The particle-particle collisions are at the origin of the transitions toward the surface modes. First, there is no transition to surface mode with one particle in the box. Second, the transition rate $\Gamma_{b \rightarrow s}$ from bulk mode to surface mode is in good agreement with the number of particle pairs $\Gamma_{b \rightarrow s} \propto N(N-1) / 2$ (see Supplemental Material, II [47]). The effect of the particleparticle collisions is to modify the orientation of the particles: if a particle-particle collision happens for a particle close to a wall, it may eventually lead a particle to contact the wall with the appropriate angle of incidence in order to transit to the surface mode (see Supplemental Material, III [47]).

The trajectories are processed to extract the root mean square displacement (RMSD) of the particles as a function of the time increment $\tau$. A first RMSD is computed for the trajectories within a smaller circular domain avoiding the boundaries. This RMSD is represented in Fig. 1(c) by a solid line. A second RMSD is computed for the trajectories close to the wall. At short times, both RMSDs show a ballistic regime for the particles moving unhindered along locally straight trajectories. For the bulk, the RMSD presents an oscillation with a first local minimum at $\tau=1.3 \mathrm{~s}$. This oscillation is a consequence of the circular motion with a natural period $\langle T\rangle=1.33 \mathrm{~s}$. The coherence of the oscillations is progressively lost at longer times, mostly because of collisions. At long times, the RMSD is subdiffusive because of finite size effects. For the wall domain, the ballistic regime gives a slightly lower velocity because the particle's velocities are reduced by the wall friction. The ballistic regime, however, extends for a longer time and the rotation of the particle in the box is observed for $\tau=8.7 \mathrm{~s}$. The first local maximum $(\tau=4.3 \mathrm{~s}$, RMSD $=0.54 \mathrm{~m})$ is consistent with the diameter of the box $(0.6 \mathrm{~m})$. With linear walls, the ballistic regime would extend for a much longer time.

The local minima for both RMSDs in Fig. 1(c) follow the same regime identified as a diffusion limit. In the absence of fluctuating trajectories, a particle would exactly come back to its initial position after a time $\langle T\rangle$. In the presence of fluctuations, the residual displacement at this local minimum is a direct measurement of the noise magnitude. This regime suggests that the origin of the noise is the same for the particles in bulk mode and the particles in surface mode.

Figure 2(a) presents a vector map of the average velocities of the particles. A color scale is used to indicate the orientation of the velocity vector with respect to the center of the box. The external wall layer shows a counterclockwise flux consistent with the trajectories of the particles in surface mode. More surprisingly, a counterflow with a clockwise flux is observed for a second inner layer. This clockwise flux can be interpreted as the consequence of the particles in bulk mode evolving in a bounded domain. In the center of the box, the particles are uniformly distributed during the acquisition and the contribution of two adjacent rotating particles cancels out. Near the wall, the particles are not able to rotate outside of the domain (see the ghost trajectory represented by a dashed line) so the contribution from two nearby particles does not cancel out systematically.

The particle transport is represented in Fig. 2(b) with a map showing the ratio $D_{\tau}(x, y) / \tau$ in which $D_{\tau}(x, y)$ is the particle displacement for a time increment $\tau=1.52 \mathrm{~s}$. This value is chosen slightly higher than the average circular period $\langle T\rangle=1.3 \mathrm{~s}$ in order to maximize the ratio between the distance traveled by the surface particles and the distance traveled by the bulk particles. A low value for $D_{\tau}(x, y) / \tau$ means that the particles are coming back to the same position after a time $\tau$ because of the circling. A large value is obtained for particles moving straight.

For most of the domain, $D_{\tau} / \tau$ is of the order of $6 \mathrm{~cm} \mathrm{~s}^{-1}$, which is significantly smaller than $v_{0}=30 \mathrm{~cm} \mathrm{~s}^{-1}$, the particles typical velocity. This low value is for the bulk populated by particles with circular trajectories, without significant transport over a time $\tau=1.52 \mathrm{~s}$. Near the walls, there is a clear difference between the two external layers identified in Fig. 2(a). For the outer layer, there is an effective transport of the particles which follows the surface streamline circulation. For the inner layer there is no increase of particle transport compared to the center of the box in contrast to what the vector map seems to indicate. This result is remarkable: it is not possible to infer the ballistic transport of particles (b) from the analysis 

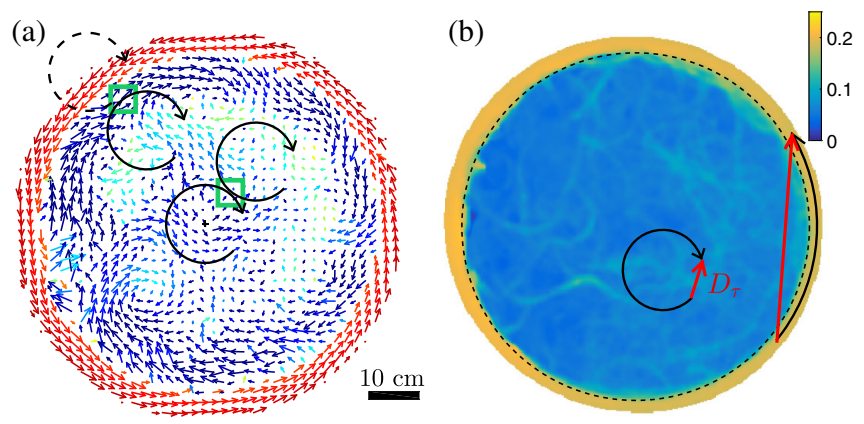

FIG. 2. (a) Average instantaneous velocity field obtained from a set of $N=7$ right-turning particles in a circular box. The color of the arrows indicates the orientation of the velocity with respect to the center of the box. Two pairs of schematic trajectories are represented on top of the vector field to illustrate the clockwise circulations. The dashed line is a nonexisting trajectory because of the wall presence. Two green boxes are represented to illustrate the trajectory sampling. (b) $D_{\tau} / \tau\left(\mathrm{m} \mathrm{s}^{-1}\right)$ with $D_{\tau}$ the distance increment during a time $\tau$. Here, the value selected for $\tau$ is $1.5 \mathrm{~s}$, close to the first minimum in the MSD of a particle in bulk mode [see Fig. 1(c)]. For a particle moving straight, $D_{\tau} / \tau$ is the instantaneous velocity of the particle. For a particle describing perfect circles with a period $T, D_{\tau} / \tau=0$ for $\tau=T$.

of the velocity map (a). The question of particle transport could also be problematic with net quantities: the integrated contribution of the velocity field in Fig. 2(a) for the inner negative layer (in blue, clockwise) and the outer positive layer (in red, anticlockwise) almost cancels. This means that one could miss the particle net transport at the walls by analyzing only the instantaneous velocity field.

A second experimental configuration is presented in Fig. 3(a). The aim of this configuration is to extract selectively the particles in two compartments according to their polarity. A set of $N_{l}=N_{r}$ particles runs in the channel with $N_{r}$ the number of particles turning right and $N_{l}$ the number of particles turning left. If a particle crosses one of the two gates and enters in a compartment, the particle is manually repositioned in the lower part of the channel. This procedure permits us to study the particle's first passage sorting rate by maintaining constant the particle number in the experiment, if one neglects the time between the gate crossing and the manual replacing of the particles (typically 2-5 s). Two series of experiments are performed, one with the experiment horizontal and the other with a small tilt of the table. Even at the lowest density explored, the particles are not in a rare collision regime. With $N=4$, there is about one collision per second, either between particles or with the walls. Consequently, there is no memory effect of the initial configuration beyond a few seconds.

Figure 3(b) presents the rate of particles entering in the target compartments. The set of experiments with the horizontal table $\left(\alpha=0^{\circ}\right)$ is for $N_{l}+N_{r}=4,8$, and 16 particles. The square data points are for the total rate of (a)
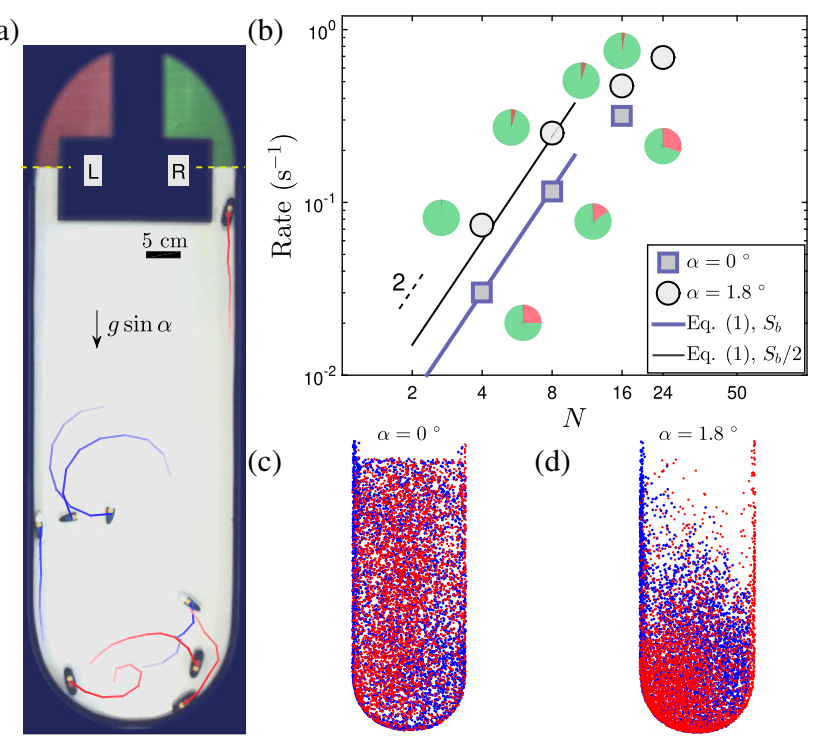

FIG. 3. (a) Top view of the sorting experiment with $N_{r}=4$ right-turning particles and $N_{l}=4$ left-turning particles. Most of the particles are in the bulk mode except for one right-handed particle that is about to reach the gate $R$ and one left-handed particle following the wall toward the gate $L$. This snapshot is for the horizontal experiment $\left(\alpha=0^{\circ}\right)$. The whole experiment can be tilted to induce a horizontal external field $g \sin \alpha$. (b) Particle extraction rate as a function of the total number of particles in the channel for the horizontal $\left(\alpha=0^{\circ}\right)$ and the tilted experiment $\left(\alpha=1.8^{\circ}\right)$. Once a particle is counted in a compartment, it is replaced in the channel. The circle charts indicate the error ratio. (c) Particle locations in the channel (right-handed red dots, lefthanded blue dots) without tilt. (d) Particle locations in the channel (right-handed red dots, left-handed blue dots) with a tilt $\alpha=1.8^{\circ}$.

particles crossing the gate $L$ and $R$, independently of their polarity. For the lowest rate measured for $N=4$, there are 16 particles entering in the compartments, which means that the relative error for this point is of the order of $1 / 15$. The expected scenario for the sorting is a collision that results in a transition from bulk mode to surface mode. Once this occurs, the particles are guided by the walls to the gates with the particles turning left or right guided to the gate $L$ or $R$, respectively (see movie v2 [47] for a successful extraction with right-turning particles). For each data point, a circle chart indicates the error ratio for the particles that went in the wrong compartment. The chart is such that no error corresponds to a green disc and an error ratio of $1: 1$ corresponds to a red disc. The error for the sorting on a horizontal table is one to two particles going in the wrong gate out of ten extracted particles. The sorting error is due to two factors: the presence of particles in the vicinity of the wrong gate because of bulk mode diffusion and the collisions close to the wrong gate (see movie v3 [47]).

In order to reduce the presence of particles in bulk mode close to the gates, a tilt of the support is imposed. Figures 3(c) and 3(d) show the effect of the tilt of the experiment on the particle locations. Each colored point is a 
trajectory point of the particles with blue dots for rightturning particles and red dots for the left-turning particles. For the horizontal experiment in Fig. 3(c), the particles are homogeneously distributed in the channel. At the curved region of the channel, there are slightly more left-turning particles (blue) on the right side and right-turning particles (red) on the left side. For the tilted experiment in Fig. 3(d) there is an increase of particle density in the bottom part of the channel at a lower altitude due to gravitaxis [50]. Contrary to the horizontal experiment, the only particles significantly represented in the upper part of the channel are the particles guided by the walls.

The second experiment with the tilted table $\left(\alpha=1.8^{\circ}\right)$ is performed with $N_{l}+N_{r}=4,8,16$, and 24 particles. First, and for all the data points, the error ratio is well below the error ratio obtained with the horizontal table. Here, 1 to 2 particles end up in the wrong gate out of 100 extracted particles. This is consistent with the map locations in Fig. 3(c) in which the only particles near the gates are the particles in the polarized wall current. Second, the total rate of extraction in Fig. 3(b) is also higher with tilt than without. This is consistent with the particle concentration that is higher with the tilted table. As discussed before, the transition from bulk mode to surface mode is only possible due to collisions between particles. Because the particle concentration is higher, the collision rate is also higher. To support this argument, one can estimate that the particle density in Fig. 3(d) is about twice the density in Fig. 3(c). This factor 2 is consistent with the factor 2 for the rates in Fig. 3(d) for $N_{l}+N_{r}=4$ and 8 .

In Fig. 3(b), the slope for the first two data points with $N=4$ and $N=8$ is compatible with a rate proportional to $N^{2}$. The power law with an exponent 2 is for a scenario in which the particles transit to the surface mode after particleparticle collisions. An estimation of the collision rate is $\mathcal{P}_{1} \times N^{2} / 2$ in which $\mathcal{P}_{1}$ is the rate collision for a pair of particles and $N^{2} / 2$ an approximation for the number of pairs. The collision rate for pairs of particles is approximated by $\left(s_{p} / S\right) \tau_{c}{ }^{-1}$ in which $s_{p}$ is the surface of a single particle, $S$ the surface occupied by the particles, and $\tau_{c}$ the coherence time of a given particle configuration. $\tau_{c}$ sets the time rate at which the particle configurations are explored and it is the only free parameter of the model. The transition rate from bulk mode to surface mode can be predicted by

$$
\Gamma=p\left(s_{p} / S\right) \tau_{c}^{-1} N^{2} / 2
$$

in which $p$ is a numerical prefactor that accounts for the fact that not all the collision will lead to a bulk to surface transition. A value of $p \approx 0.182$ is computed for the domain geometry (see Supplemental Material, V [47]). The model for the horizontal experiment in Fig. 3(b) is with $S=S_{b}$ in which $S_{b}=2.1 \times 10^{3} \mathrm{~cm}^{2}$ is the area of the channel. The model for the tilted experiment is with $S=S_{b} / 2$. The rate for the horizontal and tilted experiments is fitted with the model in Eq. (1) with $\tau_{c}=0.12 \mathrm{~s}$. This coherence time $\tau_{c}$ corresponds to a displacement for a particle $v_{0} \tau_{c}=3.7 \mathrm{~cm}$, which is consistent with the particle body length $4.5 \mathrm{~cm}$.

At large $N$, the probabilistic collision model (1) fails to describe the measured rates. This is because the number of particles in surface mode is not negligible and the effective number of particles in the bulk is less than the total number $N$. In addition, collisions between surface particles of opposite polarities tend to decrease the persistence of surface mode particles, which means that some particles in surface mode may not reach the compartments and may return in the bulk mode (see movie v4 [47]).

To summarize our work, a strategy of spontaneous sorting and extraction of active chiral particles is proposed with a strict condition of first passage through a target domain. The extraction is selective with the particle polarities and it relates to a polar ballistic transport only for the particles guided by the walls. No ballistic transport is observed for the particles away from the walls because of the circular trajectories. With a horizontal support, the particles entering in the wrong gate represent $10 \%$ to $15 \%$. This error is dominated by the particles reaching the gates by diffusion in the bulk. By slightly tilting the experiment, the error is drastically reduced because the particles in bulk almost never reach the gates by simple diffusion. With this tilt, only the particles which end up following the walls are extracted and sorted with an error of only $1 \%-2 \%$.

The particle-particle collisions could be identified as the elementary events initiating the particles sorting. This tells us how far from equilibrium an active system with circling particles is: the collisions, while being a source of orientational disorder, ultimately lead to an ordering of the particles into the two compartments.

J.S. L acknowledges support by IdEx (Initiative d'Excellence) Bordeaux and the French National Research Agency (ANR) through a project GASPP Contract No. ANR-19-CE06-0012. H. K thanks the IUF for partial funding. The authors thank R. Houques for help with the experimental setup.

[1] T. Vicsek, A. Czirók, E. Ben-Jacob, I. Cohen, and O. Shochet, Phys. Rev. Lett. 75, 1226 (1995).

[2] V. Narayan, S. Ramaswamy, and N. Menon, Science 317, 105 (2007).

[3] M. C. Marchetti, J.-F. Joanny, S. Ramaswamy, T. B. Liverpool, J. Prost, M. Rao, and R. A. Simha, Rev. Mod. Phys. 85, 1143 (2013).

[4] E. Bonabeau, M. Dorigo, D.d. R. D.F. Marco, G. Theraulaz, G. Théraulaz et al., Swarm Intelligence: From Natural To Artificial Systems (Oxford University Press, Oxford, 1999), p. 1.

[5] W. Bialek, A. Cavagna, I. Giardina, T. Mora, E. Silvestri, M. Viale, and A. M. Walczak, Proc. Natl. Acad. Sci. U.S.A. 109, 4786 (2012). 
[6] D. S. Calovi, U. Lopez, S. Ngo, C. Sire, H. Chaté, and G. Theraulaz, New J. Phys. 16, 015026 (2014).

[7] N. H. P. Nguyen, D. Klotsa, M. Engel, and S. C. Glotzer, Phys. Rev. Lett. 112, 075701 (2014).

[8] Q. Chen and B.-q. Ai, J. Chem. Phys. 143 4751, (2015).

[9] B.-q. Ai, Sci. Rep. 6, 18740 (2016).

[10] B. C. van Zuiden, J. Paulose, W. T. Irvine, D. Bartolo, and V. Vitelli, Proc. Natl. Acad. Sci. U.S.A. 113, 12919 (2016).

[11] G. Kokot, S. Das, R. G. Winkler, G. Gompper, I. S. Aranson, and A. Snezhko, Proc. Natl. Acad. Sci. U.S.A. 114, 12870 (2017).

[12] Z. Shen, A. Würger, and J. S. Lintuvuori, Soft Matter 15, 1508 (2019).

[13] X. Yang, C. Ren, K. Cheng, and H. P. Zhang, Phys. Rev. E 101, 022603 (2020).

[14] P. Liu, H. Zhu, Y. Zeng, G. Du, L. Ning, D. Wang, K. Chen, Y. Lu, N. Zheng, F. Ye et al., Proc. Natl. Acad. Sci. U.S.A. 117, 11901 (2020).

[15] S. van Teeffelen and H. Löwen, Phys. Rev. E 78, 020101(R) (2008).

[16] C. Weber, I. M. Sokolov, and L. Schimansky-Geier, Phys. Rev. E 85, 052101 (2012).

[17] P. K. Radtke and L. Schimansky-Geier, Phys. Rev. E 85, 051110 (2012).

[18] M. Mijalkov and G. Volpe, Soft Matter 9, 6376 (2013).

[19] C. Reichhardt and C. J. Olson Reichhardt, Phys. Rev. E 88, 042306 (2013).

[20] H. Löwen, Eur. Phys. J. Special Topics 225, 2319 (2016).

[21] B. Liebchen, M. E. Cates, and D. Marenduzzo, Soft Matter 12, 7259 (2016).

[22] S. Won, S. Kim, J. E. Park, J. Jeon, and J. J. Wie, Nat. Commun. 10, 1 (2019).

[23] C. Reichhardt and C. J. Olson Reichhardt, Phys. Rev. E 100, 012604 (2019).

[24] D. Levis, I. Pagonabarraga, and B. Liebchen, Phys. Rev. Research 1, 023026 (2019).

[25] C. P. Beatrici and L. G. Brunnet, Phys. Rev. E 84, 031927 (2011).

[26] J. Schwarz-Linek, C. Valeriani, A. Cacciuto, M. Cates, D. Marenduzzo, A. Morozov, and W. Poon, Proc. Natl. Acad. Sci. U.S.A. 109, 4052 (2012).

[27] C. Strandkvist, J. Juul, B. Baum, A. J. Kabla, and T. Duke, Interface Focus 4, 20140013 (2014).

[28] J. Stenhammar, R. Wittkowski, D. Marenduzzo, and M. E. Cates, Phys. Rev. Lett. 114, 018301 (2015).

[29] P. Digregorio, D. Levis, A. Suma, L. F. Cugliandolo, G. Gonnella, and I. Pagonabarraga, Phys. Rev. Lett. 121, 098003 (2018).

[30] P. Galajda, J. Keymer, P. Chaikin, and R. Austin, J. Bacteriol. 189, 8704 (2007).
[31] J. A. Drocco, C. J. Olson Reichhardt, and C. Reichhardt, Phys. Rev. E 85, 056102 (2012).

[32] A. Kaiser, H. H. Wensink, and H. Löwen, Phys. Rev. Lett. 108, 268307 (2012).

[33] N. Kumar, R. K. Gupta, H. Soni, S. Ramaswamy, and A. K. Sood, Phys. Rev. E 99, 032605 (2019).

[34] A. Costanzo, J. Elgeti, T. Auth, G. Gompper, and M. Ripoll, Europhys. Lett. 107, 36003 (2014).

[35] W. R. DiLuzio, L. Turner, M. Mayer, P. Garstecki, D. B. Weibel, H. C. Berg, and G. M. Whitesides, Nature (London) 435, 1271 (2005).

[36] E. Lauga, W. R. DiLuzio, G. M. Whitesides, and H. A. Stone, Biophys. J. 90, 400 (2006).

[37] J. Hill, O. Kalkanci, J. L. McMurry, and H. Koser, Phys. Rev. Lett. 98, 068101 (2007).

[38] P. Denissenko, V. Kantsler, D. J. Smith, and J. KirkmanBrown, Proc. Natl. Acad. Sci. U.S.A. 109, 8007 (2012).

[39] T. Ostapenko, F. J. Schwarzendahl, T. J. Böddeker, C. T. Kreis, J. Cammann, M. G. Mazza, and O. Bäumchen, Phys. Rev. Lett. 120, 068002 (2018).

[40] F. Peruani, J. Starruß, V. Jakovljevic, L. Søgaard-Andersen, A. Deutsch, and M. Bär, Phys. Rev. Lett. 108, 098102 (2012).

[41] H. Wioland, F. G. Woodhouse, J. Dunkel, J. O. Kessler, and R. E. Goldstein, Phys. Rev. Lett. 110, 268102 (2013).

[42] D. Takagi, J. Palacci, A. B. Braunschweig, M. J. Shelley, and J. Zhang, Soft Matter 10, 1784 (2014).

[43] J. Simmchen, J. Katuri, W. E. Uspal, M. N. Popescu, M. Tasinkevych, and S. Sánchez, Nat. Commun. 7, 10598 (2016).

[44] N. Tarcai, C. Virágh, D. Ábel, M. Nagy, P. L. Várkonyi, G. Vásárhelyi, and T. Vicsek, J. Stat. Mech. (2011) P04010.

[45] L. Giomi, N. Hawley-Weld, and L. Mahadevan, Proc. R. Soc. A 469, 20120637 (2013).

[46] A. Deblais, T. Barois, T. Guerin, P.-H. Delville, R. Vaudaine, J. S. Lintuvuori, J.-F. Boudet, J.-C. Baret, and H. Kellay, Phys. Rev. Lett. 120, 188002 (2018).

[47] See Supplemental Material at http://link.aps.org/ supplemental/10.1103/PhysRevLett.125.238003 for a description of the video files is given in the supplemental material file.

[48] T.-W. Su, L. Xue, and A. Ozcan, Proc. Natl. Acad. Sci. U.S.A. 109, 16018 (2012).

[49] F. Kümmel, B. ten Hagen, R. Wittkowski, I. Buttinoni, R. Eichhorn, G. Volpe, H. Löwen, and C. Bechinger, Phys. Rev. Lett. 110, 198302 (2013).

[50] B. Ten Hagen, F. Kümmel, R. Wittkowski, D. Takagi, H. Löwen, and C. Bechinger, Nat. Commun. 5, 4829 (2014).

[51] Y. Shelke, N. Srinivasan, S. P. Thampi, and E. Mani, Langmuir 35, 4718 (2019). 